\title{
'Reversible Inattention and hyperactivity in a child on Lacosamide - In south Indian Subpopulation'
}

\author{
Ismail Pasha ${ }^{1}$, Mahesh Kamate ${ }^{2}$, Suresh D K ${ }^{3}$ \\ ${ }^{1}$ Dept. of Pharmacology, KLE University's College of Pharmacy, Belgaum, Karnataka, India \\ ${ }^{2}$ Dept. of Pediatrics, KLE University, JN Medical College, Belgaum, Karnataka, India \\ ${ }^{3}$ Dept. of Pharmacology, Luqman college of Pharmacy, Gulbarga, Karnataka, India
}

\begin{abstract}
Lacosamide is a newer antiepileptic drug with a novel mode of action and is being increasingly used in children as an add-on drug in the treatment of refractory epilepsy. Its safety, efficacy and tolerance in adults, favorable pharmacokinetic profiles are encouraging its use in children. Because of lack of large studies in children the full spectrum of side effects of this drug in children is not clear. Though behavioral problems like agitation and worsening behavior have been mentioned in the earlier case reports and retrospective studies, there has been no emphasis on the inattention and hyperactivity. We here report an epileptic child on lacosamide who developed severe hyperactivity, aggression and inattention one week after starting the drug. It continued for one month till the drug was continued and the behavior then reverted to normal status after the drug was stopped. The knowledge of this important side effect and its reversibility should be considered when lacosamide is considered in a child.
\end{abstract}

Key words: Lacosamide, refractory epilepsy, inattention, hyperactivity.

\section{Introduction:}

Epilepsy is common is chronic neurological disorder in children and the main stay of the treatment is antiepileptic drugs (AED) therapy ${ }^{[1]}$. Despite availability of many of the conventional and newer antiepileptic drugs (AEDs), more than $30 \%$ of patients with epilepsy in children become refractory to current treatments. These children suffer from a poor quality of life and experience significant side effects. This has led to search for new drugs with novel mode of action, better safety and efficacy. Lacosamide is one such novel antiepileptic drug that has many characteristics of an ideal antiepileptic drug like complete oral absorption, linear pharmacokinetics, low protein binding $(<20 \%)$, long half-life, and peak blood concentration in 1-2 hours of ingestion, renal excretion, no significant drug interactions and limited hepatic metabolism. It also has a unique mode of action through slow inactivation of voltage-gated sodium channel. ${ }^{[5]}$ Encouraged by the efficacy and safety data of lacosamide in adults with epilepsy, there have been attempts to use this drug in children also. The experience is limited and only there are a few case reports and retrospective data on use of lacosamide in children in the last few years. ${ }^{[3-4]}$ We here report a five year old girl who developed significant hyperactivity as a side effect of lacosamide that persisted for one month till the drug was used and then reverted after it was stopped. This side effect has not been reported in adults and has not been emphasized in the limited literature available on use of lacosamide in children. She was a part of our prospective study to know the safety, efficacy and tolerability of lacosamide in children with refractory epilepsy.

\section{Case Presentation:}

Five year old girl, a case of cryptogenic complex partial epilepsy for last 2 years presented to us with recurrence of seizures. Her illness started as acute onset cluster of complex partial seizures, ataxia and altered sensorium for two weeks. Work up then including magnetic resonance imaging of brain, lumbar puncture, serum electrolytes, calcium, magnesium, liver and renal function tests were normal. She was treated as a case of presumed autoimmune encephalitis. She gradually improved but continued to have on and off seizures once every 2-4 weeks. She was aggressive and hyperactive after the onset of seizures which settled over 3 months' time. She failed trial of levetiracetam, clobazam, oxcarbazepine, phenytoin and carbamazepine in adequate doses. Because she had failed three conventional drugs and two newer drugs and seizures were of complex partial type we decided to start her on newer AED with novel mode of action, lacosamide after taking informed consent from the parents. We added tab lacosamide to her existing drug regimen containing carbamazepine. It was started at $25 \mathrm{mg}$ bd for one week and increased it to $50 \mathrm{mg}$ twice a day after a week (Weight-19 $\mathrm{kg}$ ). A week after she was started her on lacosamide parents noted that she had become very aggressive, abusive and hyperactive. She could not sit quiet at one place, was not listening to her parent's commands and was troubling other children at school. She was hurting her siblings and relatives, using abusive language and her sleep was also disturbed. Because of the symptoms did not subside even after 4 weeks of starting lacosamide, parents 
stopped lacosamide after 4 weeks. The behavior of the child improved after 3 days of stopping lacosamide and by the end of one week her behavior was like before.

\section{Discussion:}

Lacosamide is found to be a safe and effective antiepileptic drug in adults. The efficacy of lacosamide in group of children with refractory epilepsy was generally similar to previous reports. ${ }^{[3-4,7-9]}$ The most reported adverse events were mild to moderate and were more commonly observed during the titration phase. The most common adverse events were related to CNS and gastrointestinal side effects, and they appeared to be dose related. The treatment emergent adverse events leading to discontinuation of lacosamide were dizziness, nausea, diplopia, abnormal coordination, ataxia, vomiting, and nystagmus. ${ }^{[5,10]}$ Lacosamide appears to have a low incidence of psychiatric side effects with psychosis reported in only $0.3 \%$ of patients. Lacosamide does not cause weight gain or weight loss, neither does it change patterns in hematology, blood chemistry, and vital signs.

Based on the experience in adults, there have been attempts to use this drug in children with refractory epilepsy. Many case reports, retrospective studies and one prospective study have been published now..$^{[2-4,6]}$ Adverse events with lacosamide use in children are different than those reported in children. In a large multicenter study done on 130 children less than 16 years in Spain adverse effects were reported by patients and their families in 39 cases $(30 \%)$ which were mild, transient and in some could be managed by reduction in drug dosage. ${ }^{[2]}$ In 13 cases, the effects required cessation of lacosamide. The most prevalent adverse effects were nausea and vomiting, instability, dizziness, nystagmus, somnolence, weakness and adynamia. Other rare side effects reported in this study include anorexia, headache, insomnia, irritability, attention deficit, agitation and psychotic reaction. The behavioral side effects of lacosamide have not been emphasized in these reports/ studies. Side effects reported in the published studies on lacosamide in adults do not report any behavioural changes. ${ }^{[5]}$ Those done in children have reported irritability, behavioral outbursts, worsening behavior and agitation but none of them have emphasized on occurrence of troublesome inattention, aggressiveness and hyperactivity to the extent of prompting drug withdrawal and its reversibility after drug withdrawal which was the case in our patient. ${ }^{[3,4]}$

\section{Conclusions:}

Behavioral problems in epileptic children are very important and adversely affect the quality of life of these children. They can co-exist with epilepsy and can some time result because the antiepileptic drugs used in the management of epilepsy. Drugs like phenobarbitone, vigabatrin, gabapentin and levetiracetam are known to cause hyperactivity in a child and thus help us to avoid these drugs or closely observe children for these side effects when they are initiated on these drugs. With newer drugs the complete profile of side effects is not clear because of lack of adequate data. Our case emphasizes the occurrence and reversibility of troublesome inattention and hyperactivity following use of lacosamide.

\section{References:}

[1]. Joh SK, Hunmin K, Byung CL, Jong HC, Jiem C, Ki Joong K, Yong Seung H, Hee Hwang, Lacosamide as an adjunctive therapy in pediatric patients with refractory focal epilepsy. Brain Dev. 2013, http://dx.doi.org/10.1016/j.braindev. 2013.07.03 (Article in Press)

[2]. Casas-Fernandez C, Martınez-Bermejo A, Rufo-Campos M, Smeyers-Dura P, Herranz-Fernandez JL, Ibanez-Mico S, CampistolPlana J, et al. 2012. Efficacy and tolerability of Lacosamide in the concomitant treatment of 130 patients under 16 years of age with refractory epilepsy: A prospective, Open-label, observational, multicenter study in Spain. Drugs R D. 12, 2012, 187-197.

[3]. Gavatha M, Ioannou I, Papavasiliou AS, Efficacy and tolerability of oral lacosamide as adjunctive therapy in pediatric patients with pharmacoresistant focal epilepsy. Epilepsy \& Behavior, 20, 2011, 691-93.

[4]. Guilhoto LMFF, Loddenkemper T, Gooty VD, Rotenberg A, Takeoka M, Duffy FH, Coulter D, Urion D, Bourgeois BF, Kothare SV, Experience with lacosamide in a series of children with drug-resistant focal epilepsy. Pediatr Neurol. 44, 2011, 414-19.

[5]. Halford JJ, Lapointe M, Clinical perspectives on Lacosamide, Epilepsy Currents. 9, 2009, 1-9.

[6]. Rastogi RG and Ng YT, Lacosamide in refractory mixed pediatric epilepsy. A prospective add on study. Journal of child Neurology. 27 (4), 2012, 492-5.

[7]. Ben-Menachem E, Biton V, Jatuzis D et al, Efficacy and safety of oral lacosamide as adjunctive therapy in adults with partial-onset seizures. Epilepsia. 48, 2007, 1308-17.

[8]. Hala'sz P, Ka"lvia inen R, Mazurkiewicz-Beldzinska M et al, Adjunctive lacosamide for partial-onset seizures: efficacy and safety results from a randomized controlled trial. Epilepsia. 50, 2009, 443-53.

[9]. Chung S, Sperling MR, Biton V et al, Lacosamide as adjunctive therapy for partial-onset seizures: a randomized controlled trial. Epilepsia.51, 2010, 958-67.

[10]. Heyman E, Lahat E, Levin N, Berkovitch M, Gandelman-Marton R, Preliminary efficacy and safety of lacosamide in children with refractory epilepsy. European journal of paediatric neurology 16, 2012, 15-19. 